\title{
Determining Temporal Stability in Dominance Hierarchies
}

2

3 Vilette C. ${ }^{1,2}$, Bonnell T.R. ${ }^{1,2}$, Henzi S.P. ${ }^{1,2}$, Barrett. L ${ }^{1,2}$

4

$5 \quad{ }^{1}$ Department of Psychology, University of Lethbridge, 4401 University Drive

6 Lethbridge, Alberta, Canada, T1K 3M4

$7 \quad{ }^{2}$ Applied Behavioural Ecology and Ecosystems Research Unit, University of South Africa

10 Corresponding author:

$11 \quad$ Chloé Vilette

12 Department of Psychology, University of Lethbridge, 4401 University Drive

13 Lethbridge, Alberta, Canada, T1K 3M4

$14 \quad$ Email: c.vilette@uleth.ca

15 Phone: $+1-403-795-9191$

16

17

18

19

20

21

22

23 


\section{Abstract:}

26 The importance of social hierarchies has led to the development of many techniques for inferring

27 social ranks, leaving researchers with an overwhelming array of options to choose from. Many of

28 our research questions involve longitudinal analyses, so we were interested in a method that

29 would provide reliable ranks across time. But how does one determine which method performs

30 best?

31 We attempt to answer this question by using a training-testing procedure to compare 13 different

32 approaches for calculating dominance hierarchies (seven methods, plus 6 analytical variants of

33 these). We assess each method's performance, its efficiency, and the extent to which the

34 calculated ranks obtained from the training dataset accurately predict the outcome of observed

35 aggression in the testing dataset.

36 We found that all methods tested performed well, despite some differences in inferred rank

37 order. With respect to the need for a "burn-in" period to enable reliable ranks to be calculated,

38 again, all methods were efficient and able to infer reliable ranks from the very start of the study

39 period (i.e., with little to no burn-in period). Using a common 6-month burn-in period to aid

40 comparison, we found that all methods could predict aggressive outcomes accurately for the

41 subsequent 10 months. Beyond this 10-month threshold, accuracy in prediction decreased as the

42 testing dataset increased in length. The decay was rather shallow, however, indicating overall

43 rank stability during this period.

44 In general, a training-testing approach allows researchers to determine the most appropriate

45 method for their dataset, given sampling effort, the frequency of agonistic interactions, the

46 steepness of the hierarchy, and the nature of the research question being asked. Put simply, we 
47 did not find a single best method, but our approach offers researchers a valuable tool for

48 identifying the method that will work best for them.

50 Keywords: burn-in phase, David's score, dominance hierarchy, Elo-rating, I\&SI, longitudinal

51 data, rank dynamic, rank reliability, rank stability, training-testing approach.

53 Highlights:

54 - All ranking methods tested performed well at predicting future aggressive outcomes, 55 despite some differences in inferred rank order.

56 - All ranking methods appear to be efficient in inferring reliable ranks from the very start

57 (i.e., with little to no burn-in period), but all showed improvement as the burn-in period

58 increased.

59 - Using a common 6-month burn-in period, we found that all methods could predict

60 aggressive outcomes accurately for the subsequent 10 months. Beyond this threshold,

61 accuracy in prediction decreased as the testing dataset increased in length.

62 - Switching to a data-driven approach to assign $\mathrm{k}$-values, via the training/validation/testing

63 procedure, resulted in a marked improvement in performance in the modified Elo-rating

64 method. 


\section{INTRODUCTION}

71

73 paper. The lack of a clear answer convinced us to write it. We were conducting an analysis of

74 longitudinal data from our long-term study of vervet monkeys and wanted a method of

75 calculating dominance ranks that would give good reliability; that is, we wanted to know

76 whether the hierarchy inferred today could reliably predict a later date. If you are looking to

77 calculate dominance ranks, however, you will soon have "l'embarras du choix": there is such an

78 such an overwhelming array of options that you may not even know where to start, let alone

79 decide which one to choose. Our analyses revealed that we couldn't hope to provide a

80 straightforward answer - there is no absolute best method-but we did develop an approach that

81 enables researchers to find the method that will provide the best fit for their data. Specifically,

82 we demonstrate the use of a training-testing procedure that not only enables the most appropriate

83 method to be identified, but also offers greater insight into the structure and dynamics of a given

84 dataset. In what follows, we compare 13 different approaches for calculating dominance

85 hierarchies (seven methods, plus six analytical variants of these). We assess the methods'

86 performance, their efficiency, and the extent to which the calculated ranks obtained from the

87 training dataset reliably predict the outcome of observed aggression in the testing data set.

88

89 A Brief Survey of Analytical Methods for calculating Dominance Ranks

90

91 The analytical importance of hierarchies to our understanding of social organisation has

92 driven the development of several techniques for inferring dominance hierarchies from 
93 observational data (de Vries 1998; reviewed in Bayly, Evans, \& Taylor 2006; Whitehead 2008;

94 Briffa et al. 2013), as well as extensive research directed at their formation and maintenance (e.g.

95 Parker 1974; Dugatkin \& Earley 2004; Sasaki et al. 2016). At present, there are seven methods

96 that either have been commonly used to calculate dominance hierarchies, or newly offered as

97 improvements to existing methods.

98

Until recently, all ranking methods were based on interaction matrices. Using this

100 approach, it is possible to distinguish two ways of quantifying dominance relationships. In the

101 first, the dominance matrix is reorganized such that some numerical criterion is minimized or

102 maximized (e.g., Inconsistencies and Strength of Inconsistencies method (I\&SI): de Vries 1998;

103 ADAGIO ('approach for dominance assessment in gregarious species'): Douglas et al. 2017).

104 The second approach aims to provide a suitable measure of individual overall success, from

105 which a rank order can be derived directly (e.g. David's score: David 1987, Gammell et al. 2003;

106 Elo-rating: Elo 1978, Neumann et al. 2011). The I\&SI (method 1) and the David's score (method

107 2) are the two most commonly used ranking techniques. Despite their usefulness, they share a

108 drawback common to all matrix-based methods. Namely, they gather all observed interactions

109 within a particular time period to determine a single quasi-static ranking, with the result that the

110 obtained ratings depend directly on the number of individuals present in the matrix itself. Thus,

111 any observed fluctuation in rank across two time periods may simply be a consequence of

112 demographic events rather than variation in competitive abilities. Hence, these methods fail to

113 provide information about how ranks may change or are maintained over time.

115 In order to address actual, rather than "demographic", changes in a rank hierarchy, a 
116 common approach has been to compare hierarchies across different periods, or before and after

117 specific events, such as the integration of a new individual (Arseneau- Robar et al. 2017).

118 However, this still fails to track any potential continuous variation in the rank order of subjects

119 (c.f. Neumann et al. 2011; Newton-Fisher 2017). At the same time, breaking down the

120 assessment of rank hierarchies into shorter time windows is itself problematic, as shorter

121 sampling periods may result in a lack of data from which to infer a reliable hierarchy (Goffe,

122 Fischer \& Sennhenn-Reulen 2018). The I\&SI method has an additional downside in that it seeks

123 to produce a linear rank order, regardless of whether the society in question is expected to show a

124 linear hierarchy, or whether the data fit the assumption of linearity. These methodological flaws

125 have led to the emergence of two alternative approaches.

First, given that simple linearity is not likely to characterise the dominance structures of

128 many animal societies, Fujiii et al. (2015) developed a network-based method, called Percolation

129 and Conductance $(\mathrm{P} \& \mathrm{C})$. This permits nonlinear structure to emerge via estimates of network

130 directional consistency in the flow of dominance interactions, and the detection of blocks of

131 dominance ambiguity that are indicative of nonlinear segments of a hierarchy. This technique

132 uses paths within the agonistic network to generate an individual's probability of winning against

133 all other individuals. Despite overcoming the linearity assumption, $\mathrm{P} \& \mathrm{C}$ nevertheless follows the

134 two previous methods in being reliant on interaction matrices. This brings us to the second

135 alternative, which enables a more dynamic assessment of rank over time.

137 This method can be traced to Neumann et al. (2011), who argued that matrix-based

138 methods for rank assessment often cannot be applied to highly dynamic animal societies, or to 
139 sparse datasets. With this in mind, he introduced the Elo-rating method (method 4), derived from

140 its application in competitive games, and famous for its use in rating chess-players (Elo 1978).

141 This method enables the rating process to continue despite changes in group-composition, thus

142 circumventing one of the other drawbacks of matrix-based methods (viz. I\&SI, DS and P\&C). In

143 other words, Elo-ratings are based on the sequence in which interactions occur, with ranks

144 continuously updated. This has the advantage of allowing ratings to span changes in group-

145 composition.

147 Despite these improvements, Elo-rating also has its limitations. One fundamental

148 problem is that, in the absence of any knowledge of prior dominance relationships, the method

149 assigns all individuals the same initial Elo-rating score, which is then updated as interactions are

150 added across the observation period. Consequently, a "burn-in" period is necessary so that

151 sufficient observations can accumulate and enable the modeled rankings to catch up with the

152 computation of reliable ratings of dominance relationships (Albers and de Vries 2001; Neumann

153 et al. 2011). Both Albers \& de Vries (2001) and Neumann et al. (2011) are vague about how long

154 this process might take, probably because the duration of the burn-in will vary with the

155 frequency of agonistic interactions (Newton-Fisher 2017).

156

157 Another issue with Elo-rating is the assumption that all agonistic interactions entered into

158 the model are equivalent in their potential influence on rank trajectories. In rating subjects, the

159 variable $\mathrm{k}$ is used to determine the degree to which each interaction influences the future rank

160 trajectory of both winner and loser. In other words, it determines the number of rating points that

161 an individual gains or loses after a single encounter (Neumann et al. 2011). Newton-Fisher 
162 (2017) argues that holding k constant makes the implicit assumption that, as long as a clear

163 winner and loser can be identified, variation in the intensity of aggression does not influence

164 social dominance rank or rank trajectories.

As Elo-rating has received increasing interest, there have been a number of recent

167 attempts to address the difficulties associated with its use (e.g. Foerster et al. 2016a; Newton-

168 Fisher 2017; Goffe, Fischer \& Sennhenn-Reulen 2018; Sánchez-Tójar, Schroeder \& Farine

169 2018). This has resulted in the development of a method to calculate dominance, referred to as

170 the modified Elo-rating method (method 5). Specifically, Newton-Fisher (2017) presents two

171 developments of Neumann et al.'s (2011) R function to improve its efficiency: (i) the

172 incorporation of prior history and (ii) the recognition of differing intensities of aggression in

173 agonistic interactions. Using data from male chimpanzees (Pan troglodytes), Newton-Fisher

174 (2017) showed that incorporating even limited prior knowledge of dominance ranks substantially

175 reduced the required burn-in period and improved the effectiveness of Elo-rating in resolving

176 hierarchical structure. In addition, allowing $\mathrm{k}$ to vary further improved the Elo-rating models.

177 Taking another approach to the limitations of the burn-in period, Goffe, Fischer \& Sennhenn-

178 Reulen (2018) used "partial pooling", which rests on the assumption that all initial ratings are

179 sampled from the same distribution with a shared variation parameter $\sigma$. This Bayesian Inference

180 (BI) approach (method 6) facilitates the estimation of initial ratings, as well as the value of $k$.

Finally, one of the aims of the original Elo-rating was to track dynamic changes in rank.

183 However, most behavioural studies assume that individual dominance rank is relatively stable

184 over time (e.g. Poisbleau, Guillon \& Fritz 2010). With this in mind, Sánchez-Tójar, Schroeder \& 
185 Farine (2018) suggested a modification to the original Elo-rating based on randomizing the order

186 in which interactions occurred: the randomized Elo-rating method (method 7). They showed that

187 randomizing the order in which interactions occurred (1000 times), and estimating mean

188 individual ranks, improved performance compared to the original Elo-rating, particularly when

189 hierarchies were not unduly steep. This finding supports the idea that randomizing interaction

190 order has a beneficial effect when species' social dynamics are relatively stable and rank

191 acquisition is achieved by the pattern of maternal rank inheritance. The principles underlying all

192 seven methods are outlined in Figure 1.

193

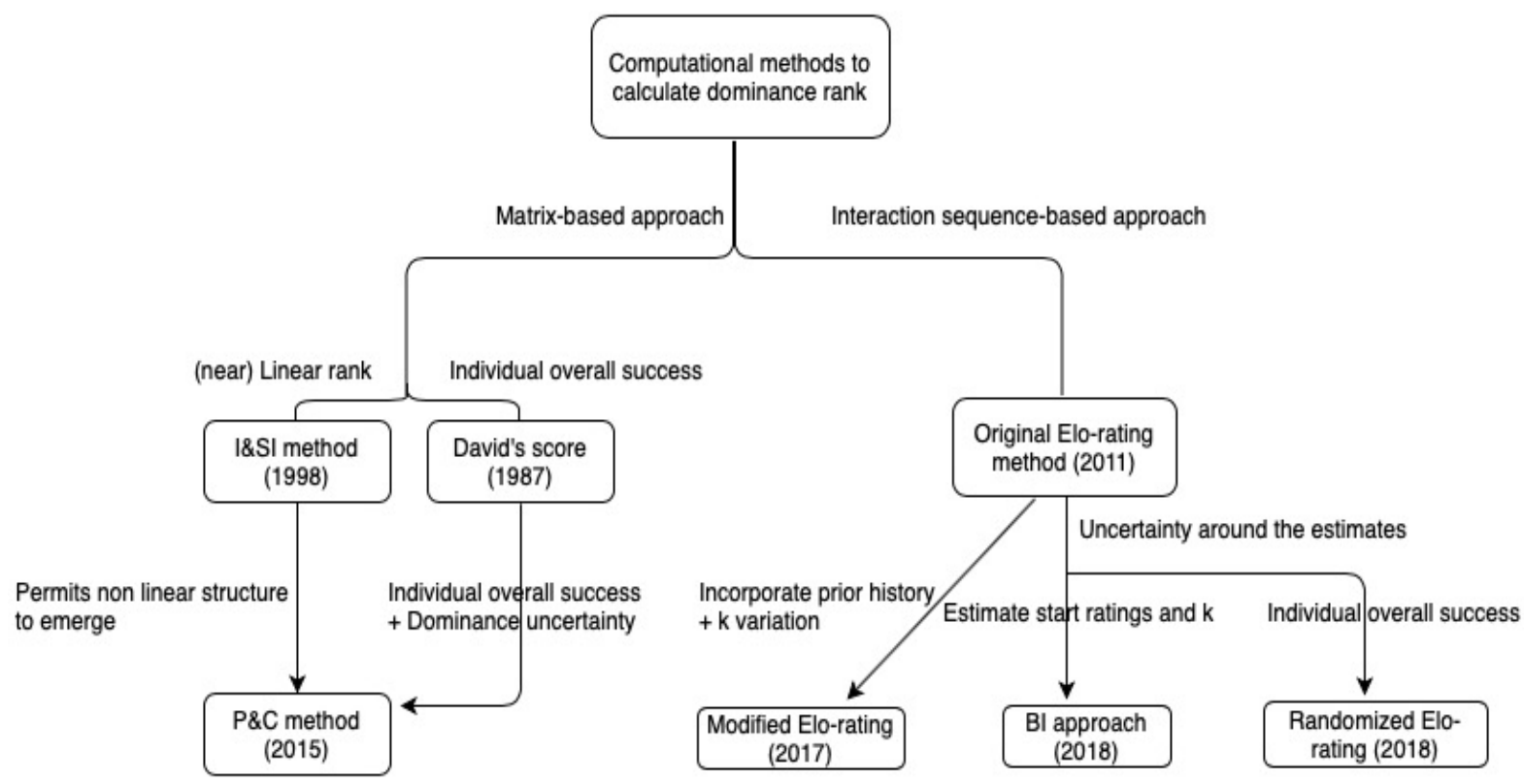

Figure 1: Main-guiding principles of each cited methods.

197 method for quantifying dominance ranks, with most focusing on comparing the level of

198 agreement among several methods when applied to real datasets (e.g. Balasubramaniam et al.

199 2013; de Vries 1998; Gammell et al. 2003; Neumann et al. 2011). Sánchez-Tójar, Schroeder \& 
200 Farine (2018) argue that, while cases where two or more methods closely match one-another

201 could signify that they are robust, it might also mean that they suffer from a common bias and, if

202 so, such comparisons can provide no information about their accuracy. Consequently, they

203 recommend simulating artificial datasets containing individuals of known rank, as well as

204 simulating interactions among those individuals under different scenarios of known steepness,

205 and then testing the validity of the method(s) by correlating the inferred hierarchy to the known

206 hierarchy.

207 Here we take an alternative, but complementary, approach. Using agonistic data from a

208 long-term study of vervet monkeys (Chlorocebus pygerythrus), we generate training-testing

209 datasets in order to determine the efficiency of the seven listed methods (along with several

210 variants of particular methods). The notion that dominance hierarchies reduce uncertainty about

211 the outcomes of contests between group members (Beaulieu et al., 2014; Mendonça- Furtado et

212 al., 2014) assumes that the state of the hierarchy at a given time will be predictive of future

213 interactions (Strauss \& Holekamp, 2019). We test this assumption by assessing whether

214 individual ranks, obtained from the training dataset, can predict the future aggressive outcomes

215 that occur in the testing dataset. We proceed as follows.

216 First, we explore each method's performance by determining the proportion of accurate

217 predictions in aggressive outcomes across the testing dataset. That is, we assess whether the

218 ranks obtained from a large training dataset are in agreement with the outcomes of aggressive

219 interactions present in our testing dataset. Given that, over time, groups undergo changes in their

220 social dynamics due to changes in the social and ecological environment, both of which may

221 underpin shifts in rank, we predict that interaction sequence-based approaches will perform 
222 better in deducing ranks than the matrix-based ones, as the former methods take into account the

223 temporal dynamics of the social hierarchy (Neumann et al. 2011; Williamson, Lee \& Curley

224 2016; Newton-Fisher 2017; Goffe, Fischer \& Sennhenn-Reulen 2018).

226 of data needed to obtain accurate estimations. A burn-in period is necessary for sufficient

227 observations to accumulate and enable a stable rank position to be calculated. However, an

228 excessive amount of data may not give a representative picture of the current state of play within

229 a group, because old, and potentially out-of-date, information will be included in the

230 calculations. In order to find the right trade-off, we determine the necessary duration of the burn-

231 in phase for each method by repeatedly reducing the length of training dataset size by 2-month

232 increments. To allow for comparison, we kept the testing dataset constant. To put this in concrete

233 terms, we maintain the same end date for the training dataset, while varying its start date. Thus,

234 as the training dataset decreases in size, only the most recent observations are included. At each

235 reduction in length, individual ranks are calculated and tested against the testing dataset. We

236 expect to see the reliability of predicted ranks to increase with the amount of data used until a

237 threshold is reached (representing the accuracy limits of older information being included in the

238 calculation), following which ranks should decrease in their reliability.

Finally, we analyze how well future aggressive outcomes can be predicted by values

240 calculated during an earlier period. To do this, we reversed the above process, keeping the

241 training dataset constant, while sequentially extending the testing dataset size by 2-month

242 increments, until we achieved a 30-month testing dataset. Our interest here was to assess the

243 length of time it would be possible to use previously calculated ranks with minimal loss of 
244 accuracy. As mentioned above, rank positions within a hierarchy can change over time due to

245 temporal variation in demographic and ecological conditions. Thus, we expect that aggressive

246 outcomes will be predicted with high accuracy initially and will then manifest a constant decay

247 as the accuracy of ranks decreases through time.

248 After investigating these seven methods, we conclude our study by proposing our own

249 modification to the modified Elo-rating that helps to improve its performance.

\section{METHODS}

a. Study site and subjects

253 part of a long-term field project at the Samara Private Game Reserve, South Africa (32o22’S,

254 24o52'E). We used data from one of our three study groups (RBM). All animals were fully

255 habituated and individually recognisable. The study group occupied semi-arid riverine woodland

256 (Pasternak et al. 2013). Group composition varied across the study period (Males: 20-6, Females:

257 13-8; Juveniles: 33-9; Infants: 11-2).

Agonistic behaviours, identities of participants and interaction outcomes were recorded

260 ad libitum on all group members (i.e., across all sex and age categories). In order to make use of

261 the most diverse and complete dataset, we included agonistic encounters with juveniles and

262 infants and also those that involved coalitions (i.e., where one or more animal comes to the aid of 
263 another against a common opponent). Unknown outcomes were discarded. Agonistic behaviours

264 included displacements, threats, chases and bites. The visibility of the habitat, together with the

265 modal presence of more than one observer (McFarland et al. 2014, Henzi at al. 2013), means it is

266 unlikely that there was any systematic bias in the recording of agonism. We recorded 11323

267 agonistic interactions between 66 individuals across the 36-month period. The initial training

268 dataset comprised 8292 interactions, with the testing dataset accounting for the remaining 3031

269 interactions.

\section{c. Methods used to infer ranks}

271 We assessed the performance of three commonly used methods for inferring longitudinal

272 hierarchies: the I\&SI method, David's scores (DS) and the Elo-rating method. We also

273 evaluated the performance of $\mathrm{P} \& \mathrm{C}$, as well as the three methods derived from Elo-ratings: the BI

274 approach, the randomized Elo-rating and the modified Elo-rating approach (see Table 1 for a

275 summary of the methods) to give a total of seven methods. As noted above, several different

276 statistical packages and options are available for the David's score and P\&C methods, thus we

277 assessed 13 methods in all.

All our calculations were implemented in R.

\begin{tabular}{|c|l|l|c|}
\hline Method & \multicolumn{1}{|c|}{ Approach } & Outcome & Analytical details \\
\hline \multirow{2}{*}{ I\&SI } & Reorder interaction matrix iteratively to & & Package: compete \\
& minimize (1) number of inconsistencies & Ordinal rank order & Function: isi13 \\
& (I) \& (2)strength of inconsistencies (SI) & & nTries $=449$ \\
\hline
\end{tabular}




\begin{tabular}{|c|c|c|c|}
\hline David's score & $\begin{array}{l}\text { Matrix based approach in which the } \\
\text { relative strength of opponents is taken } \\
\text { into account }\end{array}$ & $\begin{array}{l}\text { Individual overall } \\
\text { success determined by } \\
\text { weighting each dyadic } \\
\text { success measure by } \\
\text { the un-weighted } \\
\text { estimate of the } \\
\text { opponent's overall } \\
\text { success }\end{array}$ & $\begin{array}{l}\text { Packages: compete, } \\
\text { steepness \& EloRating } \\
\text { Indices: Pij and Dij }\end{array}$ \\
\hline $\begin{array}{l}\text { Percolation \& } \\
\text { Conductance }\end{array}$ & $\begin{array}{l}\text { Combines information from direct } \\
\text { win/loss interactions and from indirect } \\
\text { pathways to create a matrix of } \\
\text { probabilities. The network transitivity } \\
\text { determines how much to weight the } \\
\text { indirect 'wins' from these pathways }\end{array}$ & $\begin{array}{l}\text { Average dominance } \\
\text { probability }+ \\
\text { dominance uncertainty }\end{array}$ & $\begin{array}{c}\text { Package: Perc } \\
\text { MaxLength }=2 \text { and } 4\end{array}$ \\
\hline $\begin{array}{l}\text { Original Elo- } \\
\text { rating }\end{array}$ & $\begin{array}{l}\text { Scores are updated after each } \\
\text { interaction depending on the outcome. } \\
\text { Individuals start with predefine score } \\
\text { while k is a winning/losing shift } \\
\text { coefficient that is kept constant }\end{array}$ & $\begin{array}{l}\text { Individual overall } \\
\text { success }\end{array}$ & $\begin{array}{l}\text { Package: EloRating } \\
\text { Initial scores: } 1000 \\
\qquad \mathrm{~K}=100\end{array}$ \\
\hline $\begin{array}{l}\text { Modified Elo- } \\
\text { rating }\end{array}$ & $\begin{array}{l}\text { Attribute a k value to each of the } \\
\text { aggression categories }\end{array}$ & $\begin{array}{l}\text { Individual overall } \\
\text { success }\end{array}$ & $\begin{array}{c}\text { Code from } \\
\text { Newton-Fisher } 2017 \\
4 \text { categories of } \\
\text { aggression intensity. }\end{array}$ \\
\hline
\end{tabular}




\begin{tabular}{|c|c|c|c|}
\hline & & & $\begin{array}{l}\text { Lowest starting at } \mathrm{k}= \\
200 \text {, with } \mathrm{k} \text { increasing } \\
\text { by } 25 \text { per aggression } \\
\text { intensity }\end{array}$ \\
\hline $\begin{array}{l}\text { Bayesian } \\
\text { Inference }\end{array}$ & $\begin{array}{l}\text { Estimates both the most probable rank } \\
\text { order + the posterior probability of that } \\
\text { order }\end{array}$ & $\begin{array}{l}\text { Start ratings, the Elo- } \\
\text { rating winning/losing } \\
\text { shift coefficient }(\mathrm{k}) \\
\text { before computing the } \\
\text { scores }+ \text { the } \\
\text { uncertainty around the } \\
\text { estimates }\end{array}$ & $\begin{array}{l}\text { Code from Goffe, } \\
\text { Fischer \& Sennhenn- } \\
\text { Reulen } 2018\end{array}$ \\
\hline $\begin{array}{l}\text { Randomized } \\
\text { Elo-rating }\end{array}$ & Randomized interaction sequence & $\begin{array}{l}\text { Individual overall } \\
\text { success }+ \text { measure of } \\
\text { uncertainty around the } \\
\text { estimates }\end{array}$ & $\begin{array}{l}\text { Package: aniDom } \\
\text { n.rand }=1000\end{array}$ \\
\hline
\end{tabular}


We divided our dataset, using the first $80 \%(2.1 .2015-25.4 .2017)$ to train the methods, with testing undertaken on the remaining 20\% (26.4.2017 - 31.12. 2017)(Figure 2). This ensured

286 that we always had a training dataset with sufficient observations to infer reliable ranks.

287 This approach is taken from machine learning where it is a common strategy to split data into

288 training and evaluation subsets, usually with a ratio of 70-80 percent for training and 20-30

289 percent for evaluation. The two datasets are thus distinct as we are only interested in predicting

290 future outcomes and not interpolating ranks. For each method, we calculated dominance

291 hierarchies from our training data.

293 It is important to note that, in our case, each model (i.e., ranking method) is fit to the data while a

294 withheld dataset is used to assess the models. As we are interested in the reliability of rank

295 estimates across time, this approach proves to be useful with time series data as it lets us make

296 estimates of how well each model performs in forecasting (i.e., predicting future interaction

297 outcomes). Here, we make the assumption that the process model is the same between the two

298 time periods (i.e., training and testing) and we make "small world" predictions about which

299 method is best for our particular dataset.

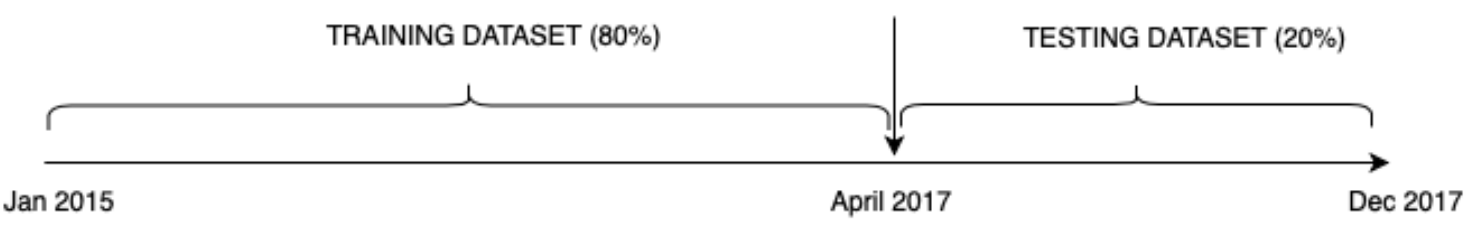


We first chose to use visualisation to examine how the rank order inferred by each

305 method differs. Given that the percentage of accurately predicted outcomes results from the

306 inferred rank order, knowing the extent to which rank order varies can help to better understand

307 each method's performance. To do so, we implemented a hierarchical clustering approach that

308 assembled our results according to their similarity. Initially, each method was assigned to its own

309 cluster. The algorithm then proceeds iteratively, at each stage joining the two most similar

310 clusters, and continuing until there is just a single cluster. In this way, methods that are most

311 similar to each other are combined into branches that are themselves fused higher up in the

312 clustering process. Euclidean distance is used to measure the dissimilarity between each pair of

313 methods. As the estimated ranks were measured on different scales, we standardized the data and

314 used the hclust R function (stats package) to generate this hierarchical clustering.

316 The obtained ranks for each method were then converted into ordinal ranks to enable

317 comparison across methods. The ordering of these ranks was used to assess how well they

318 matched the outcome of aggressive interactions for each interaction in the testing dataset (yes $=1$

319 or no $=0$ ). The proportion of accurately predicted outcomes was then translated into a percentage

320 allowing us to assess which methods (if any) outperformed others. We expected more accurate

321 predictions using the more dynamic approaches given changes in the social dynamics of our

322 study group. We excluded from analysis animals that were only present during the testing phase

323 of the dataset, but retained those individuals present only in the training dataset, as they were

324 able to provide information about their opponents. 
We assessed the methods' efficiency by estimating the necessary burn-in period. As

327 noted above, we kept our testing dataset constant while modifying the length of the training

328 dataset. Our original training dataset comprised 28 months, which we reduced sequentially by

329 two months, until only two months were left. To do so, we truncated the dataset starting from

330 January 2015 towards April 2017 (Figure 3).

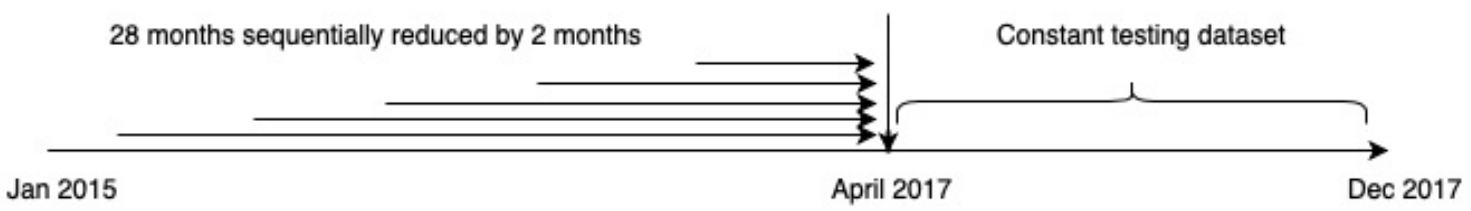

Figure 3: Modified approach used to assess the burn-in period by varying the training dataset length.

333 At each reduction in size, we computed ranks and assessed these against the testing dataset. The

334 same procedure outlined above was used to calculate the percentage of accurately predicted

335 outcomes. These percentages were then plotted in order to determine the amount of data needed 336 to predict reliable and stable ranks.

iii. $\quad$ Determining rank stability and predictability across time

341 continue to accurately predict aggressive outcomes. To do so, we gradually increased our testing

342 dataset size and looked at its impact on the percentage of accurately predicted outcomes. Based

343 on the burn-in period results (see below), we calculated the average optimal training dataset

344 length across all methods. Using the average in this way allowed us to keep the training dataset

345 constant, easing comparisons between the different methods. We then used the remaining data as

346 our testing dataset, and systematically varied its length. We began with the 2-month period that 
347 followed on directly from the training phase (July-September 2015) and then sequentially

348 increased the testing dataset by 2 months until the 30-months limit was reached in December

3492017 (Figure 4).

350

351

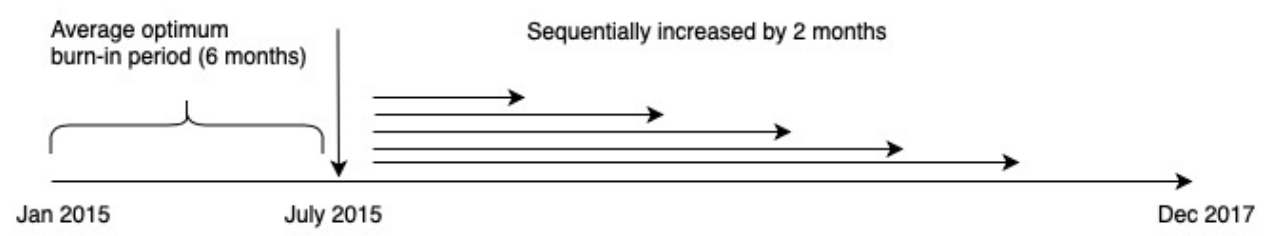

354 Ranks predicted by the training dataset were then compared to the observed outcomes in each

355 testing dataset. Percentage accuracy was plotted as a function of testing dataset length, giving us 356 an insight into the rate of decay in each method's performance.

\section{e. $\quad$ Elo-rating approach using optimized $k$}

361 categories in our dataset. As we are seeking to find the optimised k values that fit best our data,

362 we performed an optimization. We used the DEoptim package (Mullen et al. 2011). The

363 DEoptim function searches for the global optimum of the objective function (fn) between lower

364 and upper bounds on each parameter to be optimized. It is important to emphasize that the result

365 of DEoptim is a random variable, i.e., different results may be obtained when the algorithm is

366 run repeatedly with the same settings. In our case, the function fn with the highest percentage of 
367 accurately predicted outcomes was kept, along with the optimized parameters that corresponded

368 to our four different categories of threat. We assigned to these parameters the lower bound of 0

369 and the upper bound of 500. Once the optimal parameter values had been extracted, individual

370 ranks were calculated with the modified Elo-rating function provided by Newton-Fisher (2017).

371 The use of this optimization led us to modify our training/testing approach into a

372 training/validation/testing one. Specifically, we divided the original $80 \%$ training dataset in two

373 datasets, commonly called training and validation. The training dataset (i.e., the first $80 \%$ ) was

374 used to attribute $\mathrm{k}$ values, leading to the calculation of individual ranks based on these values

375 (Figure 5). The remaining 20\%, the validation dataset, allowed us to see how well these ranks

376 did in predicting the aggressive outcomes. Depending on the percentage of accurately predicted

377 outcomes, $\mathrm{k}$ values were updated accordingly in the training dataset. Once the optimised $\mathrm{k}$ values

378 were obtained, they were used to calculate the ranks from the original $80 \%$ training dataset. The

379 testing dataset then allowed us to test the efficiency of the calculated ranks in predicting future

380 aggressive outcomes.

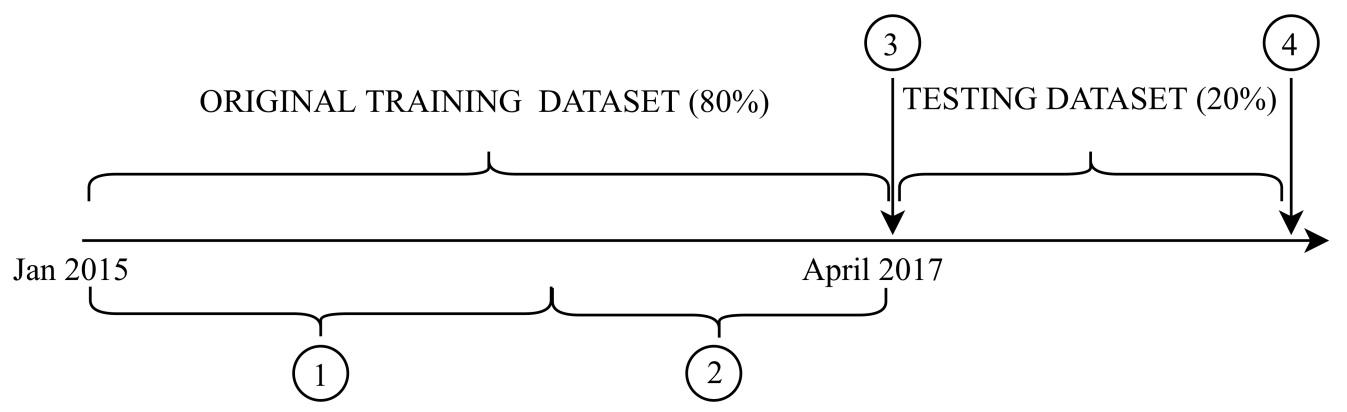

Figure 5: Modified approach used to improve the performance of the modified Elo-rating.

(1) Training dataset (80\%) used to (i) attribute $\mathrm{k}$ values to the different types of aggression (ii) calculate individual ranks.

(2) Validation dataset (20\%) used to (i) run the optimisation loop. Its outcome, the percentage of accurately predicted outcomes, will reveal how well the ranks match the aggressive outcomes and allow the loop to modify the $k$ values in order to improve the prediction.

(3) Once the optimized $\mathrm{k}$ values obtained, final ranks are computed using the original training dataset.

(4) Percentage of accurately predicted outcomes is extracted after testing the ranks on the testing dataset. 
383 All protocols were non-invasive and adhered to the laws and guidelines of South Africa and

384 Canada. Procedures were approved by the University of Lethbridge Animal Welfare Committee

385 (Protocols 0702 and 1505).

387 RESULTS

a. Which method performs best?

391 rank order in our study group (Figure 6a). The height of the fission on the vertical axis indicates

392 the similarity of ranks between two methods. The higher the height of fusion occurs, the less

393 similar the methods are in terms of their outputs. With this in mind, the output from the original

394 Elo-rating appears to be most different from the others, followed by the modified Elo-rating and

395 then the I\&SI method. The green cluster comprising the David's scores method is the most

396 similar in its outputs followed by the P\&C method and then by the BI and randomized Elo-rating

397 cluster.

399 The violin plot, which shows the percentage of accurately predicted outcomes in the testing

400 dataset, presents interesting results when combined with the dendogram (Figure 6b). While the

401 dendogram shows how similar the methods are in their outputs (rank order), the violin plots

402 show the variance in the percentage of accurate predictions produced by each method, i.e., they

403 give us a sense of the "uncertainty" in the rank outputs produced. Looking at the blue cluster (BI

404 and the randomized Elo-rating), we can see that both methods produce similar outputs (Fig 6a) 
405 and, yet, differ in their performance: the BI approach has a higher percentage of accurate

406 predictions than the modified Elo-rating (Fig. 6b). Interestingly, the blue cluster of the

$407 \mathrm{BI} /$ randomized Elo-rating and the modified Elo-rating (orange cluster) differ in their outputs (Fig

408 6a), but the randomized Elo-rating method's performance is more similar to the modified one

409 than the BI method (Fig 6b).

a.

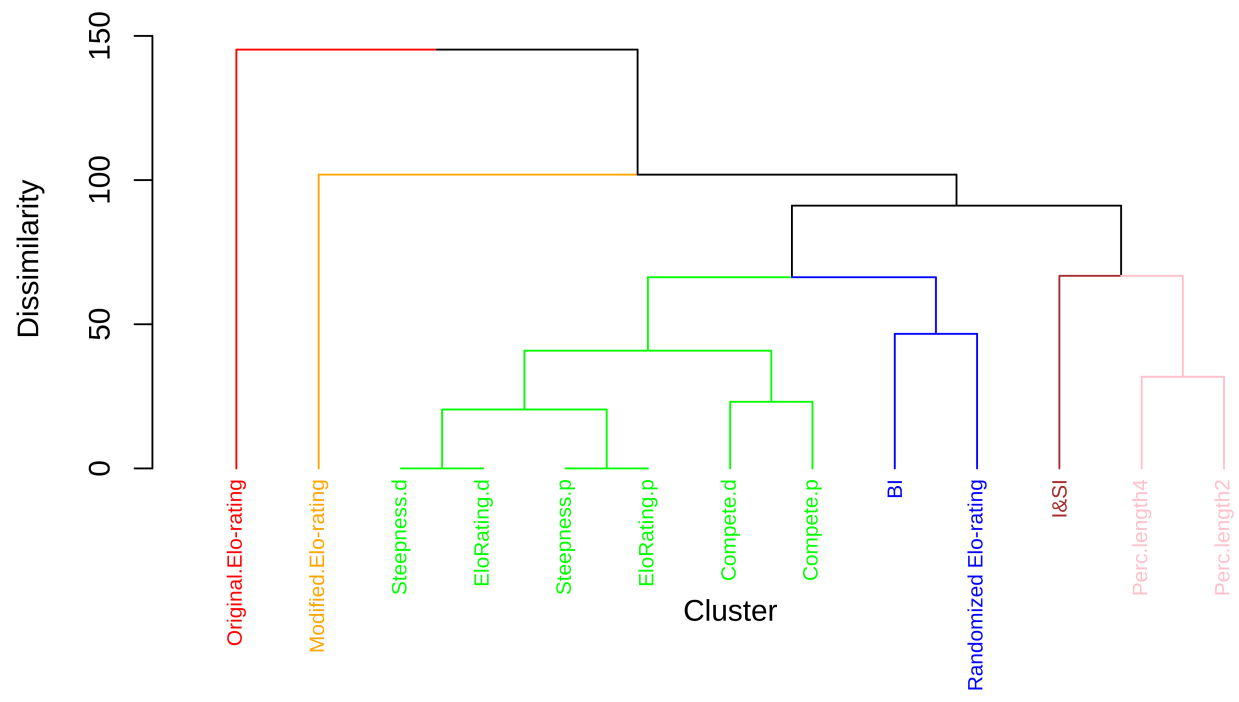

b.

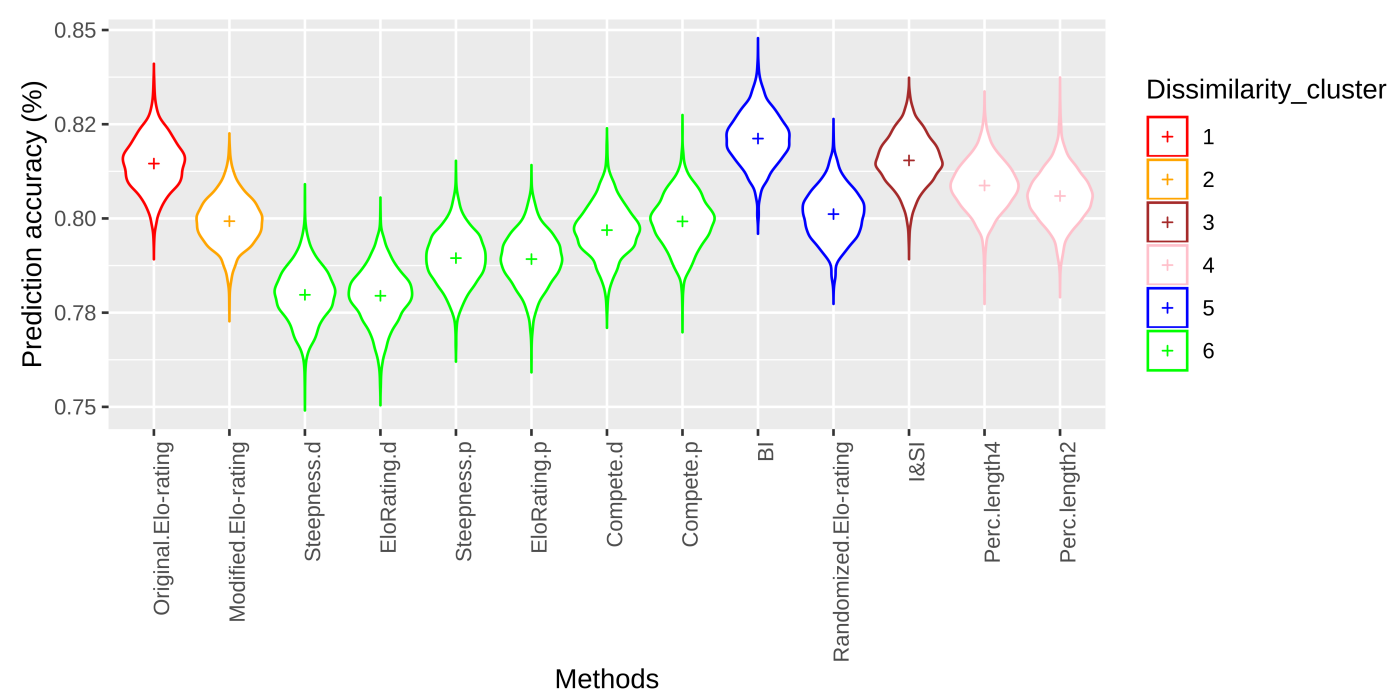


Figure 6: (a) How similar are the rank orders produced by each method? (b) How similar are the methods in their performance? Distribution of the percentage of accurately predicted outcomes across the methods used. Red, orange and blue clusters represent the interaction-based approaches while pink, brown and green ones are matrix-based methods. Green corresponds to the David's score variants.

418 These indicate that all methods performed well in inferring reliable ranks (ranging from $77.5 \%$ to

$41982.9 \%$ ). The I\&SI method provided the best fit to our data, accurately predicting $82.9 \%$ of

420 aggressive outcomes, with the BI approach producing an almost identical value of $82.6 \%$. The

421 David's score obtained from the three different packages, and via the two different functions (Dij

422 and Pij), were the lowest performing with the percentage of accurate prediction ranging from

$42377.5 \%$ to $79.9 \%$, as well as the modified Elo-rating with a percentage of predicted outcomes of

424 79.9. The David's scores from the "EloRating" package and the ones from the "steepness"

425 package (Pij and Dij function) gave the exact same percentage outcomes. Compared to Dij

426 function, the Pij predicted a higher number of accurate outcomes across all three packages used.

427 Moreover, the "compete" package with Pij has the same efficiency as the k modified Elo-rating

428 approach (79.91\%). The I\&SI method apart, the interaction-based approaches outperformed the

429 matrix-based ones. Taken together, these results show that, despite these methods differing in

430 their approach and the nature of their outputs, they all show a high level of efficiency in

431 predicting future outcomes of aggressive interactions in our dataset. 


\begin{tabular}{|c|c|c|c|}
\hline Method & Package & Option & $\begin{array}{c}\text { \% prediction } \\
\text { RBM }\end{array}$ \\
\hline $\mathrm{I} \& \mathrm{SI}$ & Compete & isi13 & 81.9 \\
\hline \multirow{6}{*}{ David's scores } & \multirow{2}{*}{ Compete } & $\mathrm{Dij}$ & 79.6 \\
\hline & & Pij & 79.9 \\
\hline & \multirow{2}{*}{ Steepness } & $\overline{\mathrm{Dij}}$ & 77.5 \\
\hline & & Pij & 78.7 \\
\hline & \multirow{2}{*}{ EloRating } & Dij & 77.5 \\
\hline & & $\overline{P i j}$ & 78.7 \\
\hline \multirow{2}{*}{$\begin{array}{l}\text { Percolance \& } \\
\text { Conductance }\end{array}$} & \multirow{2}{*}{ Perc } & maxLength4 & 81.1 \\
\hline & & maxLength2 & 80.7 \\
\hline \multirow[b]{2}{*}{ Elo-rating } & EloRating & default & 81.8 \\
\hline & $\begin{array}{c}\text { Newton- } \\
\text { Fisher's code }\end{array}$ & $\mathrm{K}$ variation & 79.9 \\
\hline $\begin{array}{l}\text { Bayesian } \\
\text { Inference }\end{array}$ & Goffe's code & Rstan & 82.6 \\
\hline $\begin{array}{c}\text { Randomized elo- } \\
\text { rating }\end{array}$ & aniDom & n.rands $=1000$ & 80.9 \\
\hline
\end{tabular}

433 Table 2: Percentage in aggressive outcome prediction over an 8-month testing dataset for our vervet monkeys' troop (RBM).

438 dataset. We expected to see that, as the length of our training dataset increased, so the percentage

439 of accurately predicted aggressive outcomes should also increase. However, as the training 
440 dataset begins to accumulate older aggressive interactions, we expected the accuracy of the

441 percentage of predicted outcome to decrease. This assumption was borne out by our results to a

442 certain extent (Figures 7 and 8). For the most part, however, accuracy of predicted outcomes was

443 not greatly affected by the length of the training dataset.

444 For ease of presentation and interpretation, we chose to look at the dynamic and static

445 approaches separately. Figure 7 presents the dynamic approaches and shows that the length of

446 the training dataset does not have any impact on three of the four methods: the original Elo-

447 rating, the BI and the modified Elo-rating. In fact, these three methods perform very well

448 regardless of the length of the training dataset. There is some evidence to suggest, however, that

449 the BI approach and original Elo-rating method produce slightly better predictions once the

450 training dataset has exceeded four months. As for the randomized Elo-rating method, this shows

451 a more acute sensitivity to the amount of data in the training set. A first peak in accurate

452 prediction appears at training set lengths between 4 and 8 months. Past 8 months, the accuracy in

453 outcome prediction decreases as the training dataset length increases. 


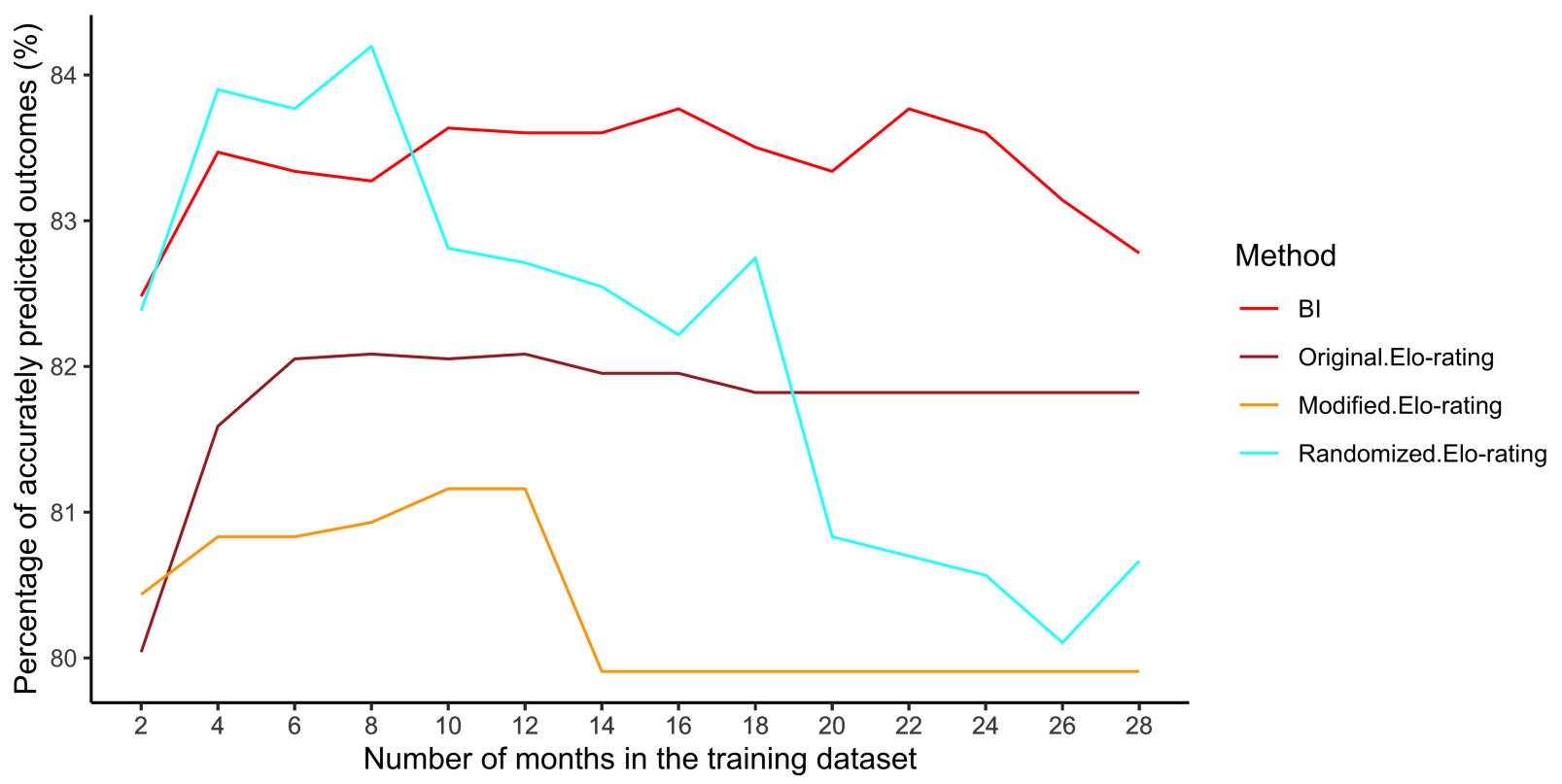

$455 \quad$ Figure 7: Variation of the percentage of outcome prediction with the dynamic approaches in function the number of months included in the training dataset.

459 accurately predicted outcomes depending on the length of the training dataset. Despite this

460 sensitivity, a maximum in prediction accuracy can be found for each of these methods. On

461 average, these peaks occur at 6 months, which we suggest represents the optimal length of burn-

462 in period needed to accurately predict future outcomes in the data. As the number of months in

463 the training dataset increases, we also see more of a decay in accurate prediction compared to the

464 more dynamic methods (as one would expect). Moreover, the I\&SI method displays a higher

465 percentage of accurately predicted outcomes compared to the dynamic methods when the

466 training dataset spans the period of 2 to 12 months. 


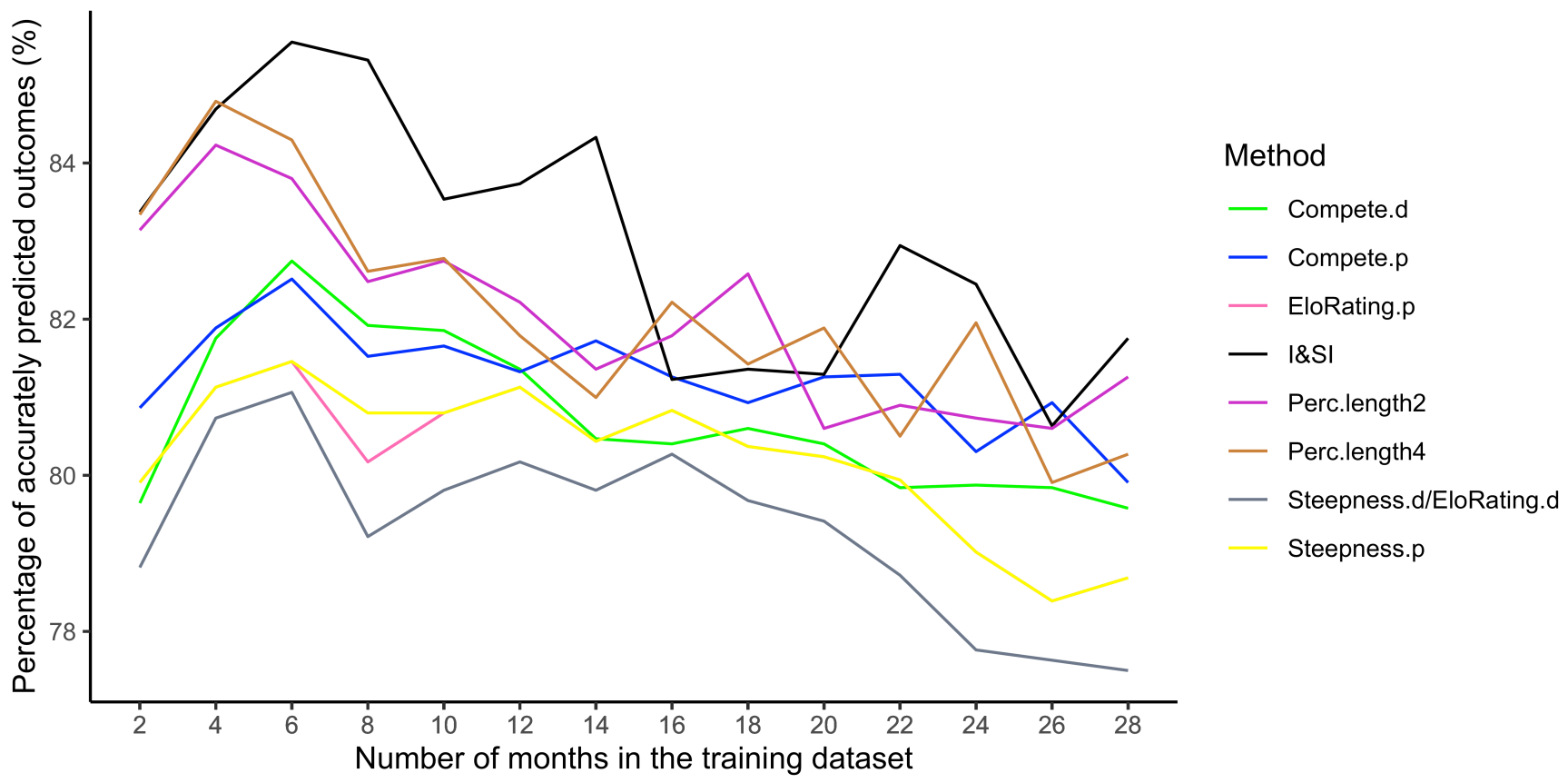

Figure 8: Variation of the percentage of outcome prediction with the static approaches in function the number of months included in the training dataset.

472 average optimal burn-in period obtained from the previous analysis. With the exception of P\&C,

473 all methods from the static approaches had an optimal percentage of accurate prediction with a 6-

474 month burn-in period. The dynamic approaches reached saturation sooner, between 2 and 4

475 months, the randomized Elo-rating being the exception with an 8-month burn-in period. We

476 therefore used a 6-month burn-in period as this represents the best compromise in terms of

477 enabling comparison across all methods. Shortening the burn-in period in this manner this

478 manner gave us a larger testing dataset of 30 months in total (2.5 years). 
479 The percentage of accurate predictions for each testing dataset length is plotted in Figure 9a-c.

480 All methods show the same pattern. First, a decline in outcome predictability occurs at 4 months,

481 which is then followed by a peak in prediction accuracy corresponding to a testing dataset of 8-

48210 months in length (Fig.9a). Past 10 months, the accuracy of predicted outcomes shows a

483 constant and slow decay. These fluctuations are more or less marked depending on the method

484 used. This pattern (i.e., where the curve's fluctuation is more marked) is picked up by all

485 dynamic approaches, while some of the static ones (I\&SI and P\&C) display the same pattern in

486 fluctuations for the first 10 months, but this is much less pronounced. The I\&SI, as well as the

487 P\&C approach, stand out as the methods producing a set of ranks that will lead to the highest

488 percentage of accurately predict outcomes over the whole testing dataset's length (Figure 9.a),

489 followed by the BI approach that presents an overall slower decay in prediction. The rest of the

490 methods are clustered with a lower percentage of accurately predicted outcomes throughout the

491 testing dataset's length.

In order to examine these patterns in more detail, we separated the dynamic and static

493 approaches (Fig. 9 b and c). With respect to dynamic approaches, as mentioned above, the BI

494 and the original Elo-rating display the general pattern described above. The randomized and the

495 modified Elo-rating show lower performance overall but, following the peak in prediction

496 accuracy at 10 months, the accuracy of the randomized Elo-rating then remains constant, while

497 the modified Elo-rating produces an increasing percentage of accurately predicted outcomes as

498 the length of the testing dataset increases. With regard to the static approaches, both the I\&SI and

499 P\&C methods display the same pattern as the BI and the original Elo-rating. The David's score

500 shows a smoother curve, with a constant decrease in accuracy once past a testing dataset of 2

501 months. The "compete" package appears to perform better than the "steepness" and "EloRating" 
502 packages, which produce equivalent curves. Finally, the I\&SI and P\&C methods display a

503 higher percentage of accurately predicted outcomes compared to the dynamic methods

504 throughout the whole testing dataset length, except for P\&C with maxLength 2 past 26-months

505 of the testing dataset.
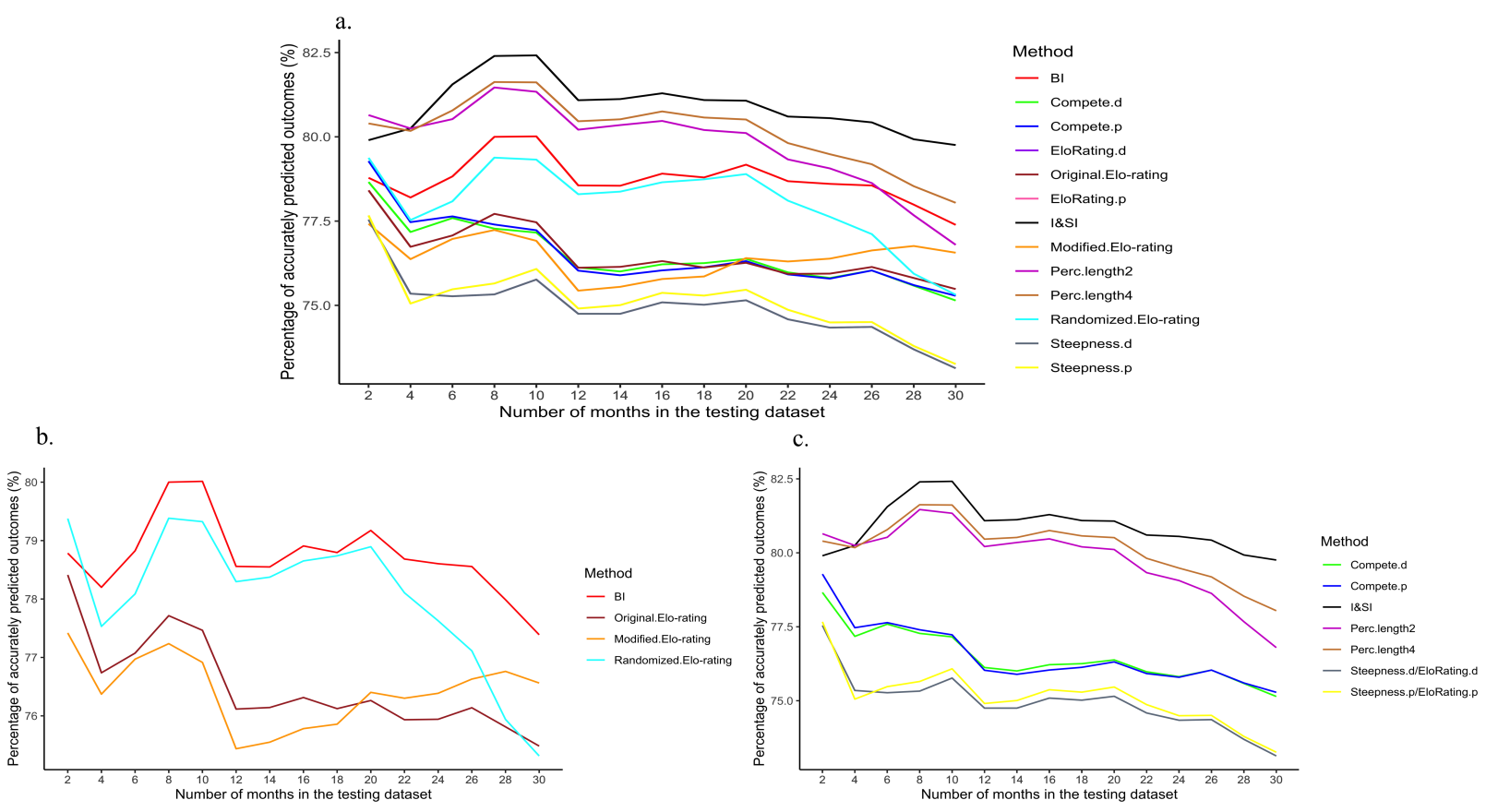

507 Figure 9: Variation of the percentage of accurate predictions as a function of testing dataset

508 length across (a) all methods, (b) dynamic approaches only, (c) static approaches only.

511 obtain the best-maximised $\mathrm{k}$ values leading to the calculation of accurate ranks. The values

512 attributed to the aggression categories are represented in Table 3. 


\begin{tabular}{|c|c|c|}
\hline & Modified Elo-rating & Alternative k values \\
\hline Physical aggression & 300 & 47.8 \\
\hline Active aggression & 250 & 33.4 \\
\hline Stationary aggression & 225 & 43.1 \\
\hline Non-aggressive & 200 & 31.4 \\
\hline
\end{tabular}

514 Table 3: k values attributed to the four types of aggressive categories for the two different

methods

516 With these new k values, $84.3 \%$ of accurately predicted outcomes were obtained over the 8-

517 month testing dataset. This alternative approach thus offers the best performance among all the

518 tested methods. The use of the optimisation enabled us to increase its performance by nearly $5 \%$.

\section{DISCUSSION}

522 methods performed well. Given this, it seems tempting to conclude that it is possible to simply

523 flip a coin or otherwise choose a method randomly. This would be the wrong conclusion,

524 however, as our analyses forced us to the realisation that the best method will always be a

525 dataset-specific phenomenon. The true value of our analyses lies in the demonstration that a

526 training-testing procedure provides researchers with the tool they need to guide their choice of

527 method. In other words, although we have failed to identify the best method, we have generated

528 a means by which researchers can find the best method for them. 
More specifically, the training-testing procedure we used here not only enables

530 informative comparisons to be made between different methods, but also gives researchers

531 important insights into the length of the burn-in period needed to produce accurate rank

532 predictions, as well as the future duration over which a given set of ranks can be used in analysis.

533 In this way, researchers can determine which will be the most appropriate method, given

534 sampling effort, the frequency of agonistic interactions, the steepness of the hierarchy, and the

535 nature of the research question being asked.

536 The use of this procedure also allows researchers to probe the social dynamics of their

537 study species in more depth. For example, when comparing ranks and outcomes, our assumption

538 was that any mismatches represented a rank being wrongly attributed. However, it would be

539 interesting (and possible) to look at the outcomes of aggressive encounters that do not match the

540 ranks assigned to each participant, when both were extracted on the same day. This would give

541 us a better idea of the true degree of outcome unpredictability, allowing us to assess whether

542 uncertainty in rank assignment is due to the nature of the aggressive interaction itself or whether

543 it reflects something about the context in which it takes place. Indeed, in cases of high outcome

544 predictability and rank stability (i.e., where rank challenges would seem unprofitable), it may be

545 the initiation and/or escalation of aggressive interactions that is unpredictable and linked to the

546 broader ecological and social context. The procedure can also be used to assess the rate at which

547 immature animals integrate into the adult hierarchy. In other words, the training/testing approach

548 enables researchers to investigate dynamic pattern of hierarchy formation and maintenance, and

549 is not simply a means of determining the most appropriate ranking method. Indeed, it can also be

550 used to investigate temporal aspects of the aggressive outcome prediction at a dyadic level,

551 rather than the whole group, and could thus offer a better understanding of the detailed social 
552 dynamics of a given study species. Another way to look at social dynamics is offered by Strauss

553 \& Holekamp (2019) who propose the concept of a longitudinal hierarchy to estimate the

554 dynamics $(\Delta)$ of dominance hierarchies.

555 More generally, our findings demonstrate how researchers can use the training-testing

556 procedure to choose the most appropriate method for their needs, given the amount of data they

557 have at their disposal, and the nature of their study question. We acknowledge, however, that the

558 use of empirical data does not allow us to distinguish between the two sources of error that could

559 explain the methods' performance: (i) inadequacies of the method, and (ii) real biological

560 change. Thus our findings on how different methods compare are only valid with respect to our

561 data and cannot be assumed to apply to other datasets. Within our dataset, we feel it is safe to

562 assume that variation in a method's performance compared to others reflects something about the

563 method itself, given that we tested all methods on the same training/testing datasets such that any

564 biological changes within the dataset should have be detected by all methods equally.

565 This is where the usefulness of simulated data comes into play as this allows one to tease

566 apart these two sources of error more effectively, as well as gaining some more general insights

567 into each method (Sánchez-Tójar, Schroeder \& Farine, 2018). For example, Goffe, Fischer \&

568 Sennhenn-Reulen's (2018) advocate running simulation approaches to test how well Elo-ratings

569 reflect the (simulated) true structure and how sensitive the rating is to true changes in the

570 hierarchy. Nevertheless, we believe the training-testing procedure is valuable empirically for

571 those interested in determining how much the methods vary when run on the exact same dataset,

572 and why this might be. It is also the case that, although simulated data provide insight into how

573 well a given method is able to recover the true hierarchy, simulated data are also much tidier and

574 "well behaved" compared to actual field data, so one should still be cautious about proclaiming 
575 any one method better than the others, as it may be sensitive to qualities of empirical data that do

576 not occur in simulations.

With respect to our specific dataset, our main finding is that all methods tested performed

579 well, despite some differences in inferred rank order. With respect to the required burn-in period,

580 again, all methods appear to be efficient in inferring reliable ranks from the very start (i.e., with

581 little to no burn-in period), but all showed improvement as the burn-in period increased. In

582 general, we found that dynamic methods were less sensitive to the amount of data present in the

583 training dataset compared to the static approaches. As a result, dynamic approaches show a

584 constant efficiency regardless the length of the training dataset compared to the static

585 approaches. This is not unexpected given that methods dynamic approaches track rank variations

586 continuously and update the ranks after each interaction. We should also highlight that, when the

587 training dataset did not exceed 12 months, it was the static I\&SI and P\&C methods that produced

588 the highest percentage of accurately predicted outcomes.

590 When we adopted a common 6-month burn-in period, all methods could predict

591 aggressive outcomes accurately for the subsequent 10 months. Past this 10-month threshold,

592 however, accuracy in prediction decreased as the testing dataset increased in length, but the

593 decay was rather shallow, and there was a high predictability in aggressive outcomes across time,

594 indicating overall rank stability during this period. This is not to say, however, that rank

595 predictability did not fluctuate as the length of the training period increased, with the degree of

596 fluctuation was dependent on the length of the method used. This suggests that real rank shifts

597 were occurring in the study group during particular periods, and that the training-testing 
598 procedure provides a way to home in on such periods of instability. The combination of high

599 overall predictability in aggressive outcomes with some temporal fluctuation suggests that, in our

600 study species, rank order shows a form of "regression to the mean". That is, individuals may

601 experience very mild shifts in relative rank position up or down the hierarchy across time, but

602 nevertheless occupy more or less the same "absolute" position in the hierarchy. This, in turn,

603 suggests that rank changes may reflect the ecological and demographic contexts in which they

604 occur, rather than pointing to genuine changes in inherent power. In line with this interpretation,

605 the dynamic methods produce more fluctuations than the static methods: the former are likely to

606 catch small shifts in rank position as they constantly update, whereas the static methods, which

607 combine and cluster interactions across time, are more likely to produce a rank order that fits the

608 overall social dynamic.

610 It is also important to remember that we converted the output from each method to ordinal ranks

611 to facilitate comparison, and differences between (the various kinds of) Elo-ratings can be

612 absolutely very small. Such differences would lead to a shift in calculated rank order and hence

613 to fluctuation in prediction accuracy, but, in reality, may have no discernable impact on an

614 individual's social standing in the group. That is, such fluctuation may, in effect, represent noise

615 around a stable rank order. Even though Strauss \& Holekamp (2019) recommend the use of

616 ordinal rankings for identifying hierarchy dynamics, this point thus serves to highlight a

617 limitation of our study in that we looked only at rank order and did not consider the magnitude of

618 rank differences nor any uncertainty around rank calculations. These components may very well

619 matter, especially in species where a linear rank order may not be representative of the social

620 hierarchy. This point also serves to highlight the true advantage of methods like the BI, 
621 randomized Elo-rating and the $\mathrm{P} \& \mathrm{C}$ approach, which enable researchers to look at the

622 uncertainty around ranks, and thus gain a better understanding of the social hierarchy.

625 of certain methods. For example, the randomized Elo-rating approach was developed to enhance

626 performance in societies where ranks remain fairly stable across time (Sánchez-Tójar, Schroeder

627 \& Farine 2018) — a situation that, as noted above, pertains in our study group. Under such

628 conditions, the sequence in which interactions occur should not affect the inferred ranks. Our

629 results help validate this assumption. When the training data set was short (i.e., 6 months), the

630 sequence of interactions clearly did not matter, and the method gave good results. As the training

631 dataset increased up to 28 months, however, there was a decline in accuracy, which no doubt

632 reflects the fact that, over this period, demographic change was inevitable and the order of

633 interactions may well have begun to exert an influence on the structure of the hierarchy. Given

634 this outcome with our data (and assuming this holds true across other datasets), this suggests that

635 the randomised Elo-rating method will also prove useful in determining when and where

636 interaction order matters in a given dataset. Indeed, Sánchez-Tójar, Schroeder \& Farine (2018)

637 suggest that researchers can control for factors such as changing ranks or winner-loser effects by

638 controlling which parts of the data are randomised. For example, researchers interested in

639 tracking how individual ranks change across days could increase the accuracy of the daily rank

640 estimates by randomizing observations within each day.

641

642 Our own modification to the Elo-rating also provided some insight into the method's

643 workings. We produced an increase of nearly 5\% in accurately predicted outcomes compared to 
644 Newton-Fisher's (2017) modified Elo-rating. Newton-Fisher's (2017) argument was that

645 allowing $\mathrm{k}$ to vary would improve accuracy as the specific nature of aggression could affect the

646 outcome and impact of aggressive encounters. Our results did not support this, but we reasoned

647 that this was most likely due to the fact that our values of k were assigned arbitrarily. Switching

648 to a data-driven approach to assign k-values, via the training/validation/testing procedure,

649 resulted in a marked improvement in performance. Thus, the type of aggression shown does, in

650 fact, matter to predictive accuracy, but arbitrarily assigning values to aggression types may fail to

651 represent its importance correctly. It is also worth noting that we did not investigate the extent to

652 which k-values, and hence ranks, were affected by the relative length of the training and testing

653 periods used in the optimisation procedure (i.e., we used only a 80:20 split), nor did we consider

654 any potential variation in values of $\mathrm{k}$ through time (e.g., due to seasonal effects). More work is

655 needed to understand better the impact of relative lengths of training and testing periods, as well

656 as the stability of $\mathrm{k}$ values through time.

657

658 In conclusion, we suggest that the insights into group dynamics provided by a training-

659 testing procedure, combined with the ability to determine which ranking method is best suited to

660 a given dataset, far outweigh the time costs associated with running the procedure. A training-

661 testing approach gives researchers the power to tailor the selection of a dominance-ranking

662 method to the particular nature of the dataset they are using, allowing them to take into account

663 sampling effort, frequency of agonistic interactions and the steepness of the hierarchy, as well as

664 the particular kinds of analyses they wish to conduct. In addition, comparison of methods and the

665 dynamic patterns they reveal further enable researchers to home in on regions of their dataset 
666 that will permit analyses into how and why rank shifts occur and discover the underlying causes

667 of both rank stability and unpredictability across time.

669 Acknowledgments:

670 We thank Mark and Sarah Tompkins for the permission to work at Samara, Kitty and Richard

671 Viljoen for their continued logistic support. We thank Damien Farine and Alfredo Sánchez-Tójar

672 for helpful discussions as well as for pointing out an error in the rank's computation with the

673 randomized Elo-rating method. We are also very grateful to the many research assistants who

674 contributed to the database, as well as our amazing monkeys (and George), without which only

675 simulated data would be available.

676 The research was supported by NSERC Discovery (Canada) grants to LB and SPH and by the 677 Canada Research Chairs program to LB, TRB and CV.

678

679 References:

680

681 Albers, PC, \& Vries, HD (2001). Elo-rating as a tool in the sequential estimation of dominance

682 strengths. Animal Behavior, 489-495.

683 Arseneau-Robar, T. J. M., Taucher, A. L., Schnider, A. B., van Schaik, C. P., \& Willems, E. P.

684 (2017). Intra-and interindividual differences in the costs and benefits of intergroup aggression in

685 female vervet monkeys. Animal behaviour, 123, 129-137.

686 Balasubramaniam, K.N., Berman, C.M., De Marco, A., Dittmar, K., Majolo, B., Ogawa, H., 687 Thierry, B. and De Vries, H., (2013). Consistency of dominance rank order: a comparison of 
688 David's scores with I\&SI and Bayesian methods in macaques. American journal of

689 primatology, 75(9), pp.959-971.

690 Bayly, K. L., Evans, C. S., \& Taylor, A. (2006). Measuring social structure: a comparison of

691 eight dominance indices. Behavioural Processes, 73(1), 1-12.

692 Briffa, M., Hardy, I. C., Gammell, M. P., Jennings, D. J., Clarke, D. D., \& Goubault, M. (2013).

693 Analysis of animal contest data. Animal contests, 379.

694 David, H. A. (1987). Ranking from unbalanced paired-comparison data. Biometrika, 74(2), 432-

695436.

696 De Vries, H. A. N. (1998). Finding a dominance order most consistent with a linear hierarchy: a

697 new procedure and review. Animal Behaviour, 55(4), 827-843.

698 Douglas, P. H., Ngomo, A. C. N., \& Hohmann, G. (2017). A novel approach for dominance

699 assessment in gregarious species: ADAGIO. Animal Behaviour, 123, 21-32.

700 Dugatkin, L. A., \& Earley, R. L. (2004). Individual recognition, dominance hierarchies and

701 winner and loser effects. Proceedings of the Royal Society of London. Series B: Biological

702 Sciences, 271(1547), 1537-1540.

703 Elo, A. E. (1978). The rating of chessplayers, past and present. Arco Publications.

704 Foerster, S., Franz, M., Murray, C. M., Gilby, I. C., Feldblum, J. T., et al. (2016). Chimpanzee

705 females queue but males compete for social status. Scientific Reports, 6, 35404.

706 Fujii, K., Jin, J., Shev, A., Beisner, B., McCowan, B., \& Fushing, H. (2015). Perc: using

707 percolation and conductance to find information flow certainty in a direct network. R Package

708 Version 0.1 . 
709 Fushing, H., McAssey, M. P., \& McCowan, B. (2011). Computing a ranking network with

710 confidence bounds from a graph-based beta random field. Proceedings of the Royal Society A:

711 Mathematical, Physical and Engineering Sciences, 467(2136), 3590-3612.

712 Gammell, M. P., Vries, H. D., Jennings, D. J., Carlin, C. M., \& Hayden, T. J. (2003). David's

713 score: a more appropriate dominance ranking method than Clutton-Brock et al.'s index. Animal

714 behaviour, 66(3), 601-605.

715 Goffe, A. S., Fischer, J., \& Sennhenn-Reulen, H. (2018). Bayesian inference and simulation

716 approaches improve the assessment of Elo-ratings in the analysis of social behaviour. Methods in

717 Ecology and Evolution, 9(10), 2131-2144.

718 Henzi, S. P., Forshaw, N., Boner, R., Barrett, L., \& Lusseau, D. (2013). Scalar social dynamics

719 in female vervet monkey cohorts. Philosophical Transactions of the Royal Society B: Biological

720 Sciences, 368(1618), 20120351.

721 McFarland, R., Barrett, L., Boner, R., Freeman, N. J., \& Henzi, S. P. (2014). Behavioral

722 flexibility of vervet monkeys in response to climatic and social variability. American journal of

723 physical anthropology, 154(3), 357-364.

724 Mullen, K., Ardia, D., Gil, D. L., Windover, D., \& Cline, J. (2011). DEoptim: An R package for

725 global optimization by differential evolution. Journal of Statistical Software, 40(6), 1-26.

726 Neumann, Christof, Julie Duboscq, Constance Dubuc, Andri Ginting, Ade Maulana Irwan,

727 Muhammad Agil, Anja Widdig, and Antje Engelhardt. (2011) Assessing dominance hierarchies:

728 validation and advantages of progressive evaluation with Elo-rating. Animal Behaviour 82, no. 4

729 (2011): 911-921.

730 Newton-Fisher, N. E. (2017). Modeling social dominance: Elo-ratings, prior history, and the

731 intensity of aggression. International journal of primatology, 38(3), 427-447. 
732 Parker, G. A. (1974). Assessment strategy and the evolution of fighting behaviour. Journal of

733 theoretical Biology, 47(1), 223-243.

734 Pasternak, G., Brown, L. R., Kienzle, S., Fuller, A., Barrett, L., \& Henzi, S. P. (2013).

735 Population ecology of vervet monkeys in a high latitude, semi-arid riparian

736 woodland. Koedoe, 55(1), 01-09.

737 Poisbleau, M., Guillon, N., \& Fritz, H. (2010). Preservation of winter social dominance status in

738 Brent Geese Branta bernicla bernicla within and across winters. Journal of Ornithology, 151(3),

$739 \quad 737-744$.

740 D. R. (2018). A practical guide for inferring reliable dominance hierarchies and estimating their

741 uncertainty. journal of animal ecology, 87(3), 594-608.

742 Sasaki, T., Penick, C. A., Shaffer, Z., Haight, K. L., Pratt, S. C., \& Liebig, J. (2016). A simple

743 behavioral model predicts the emergence of complex animal hierarchies. The American

$744 \quad$ Naturalist, 187(6), 765-775.

745 Strauss, E. D., \& Holekamp, K. E. (2019). Inferring longitudinal hierarchies: Framework and

746 methods for studying the dynamics of dominance. Journal of Animal Ecology, 88(4), 521-536.

747 Whitehead, H. (2008). Analyzing animal societies: quantitative methods for vertebrate social

748 analysis. University of Chicago Press.

749 Williamson, C. M., Lee, W., \& Curley, J. P. (2016). Temporal dynamics of social hierarchy

750 formation and maintenance in male mice. Animal behaviour, 115, 259-272

751

752 Appendix analyses for each method. 
757 of iterations (nTries). To do so, we performed an optimization to find the nTries that best fit our

758 data. We used the DEoptim package (Mullen et al. 2011). The DEoptim function searches for

759 the global optimum of the objective function ( $\mathrm{fn}$ ) between lower and upper bounds on each

760 parameter to be optimized. It is important to emphasize that the result of DEoptim is a random

761 variable, i.e., different results may be obtained when the algorithm is run repeatedly with the

762 same settings. In our case, the function fn with the highest percentage of accurately predicted

763 outcomes was kept, along with the optimized parameter corresponding to the number of

764 iterations (nTries). We assigned to this parameter the lower bound of 50 and the upper bound of

765 1000. Once the optimal parameter value had been extracted, individual ranks were calculated

766 using the latest function version "isi13" from the R package "compete".

767 The use of this optimization led us to modify our training/testing approach into a

768 training/validation/testing one. Specifically, we divided the original $80 \%$ training dataset in two

769 datasets, commonly called training and validation. The training dataset (i.e., the first $80 \%$ ) was

770 used to attribute the nTries value, leading to the calculation of individual ranks based on this

771 value. The remaining $20 \%$, the validation dataset, allowed us to see how well these ranks did in

772 predicting the aggressive outcomes. Depending on the percentage of accurately predicted

773 outcomes, the nTries value was updated accordingly in the training dataset. Once the optimised

774 nTries value was obtained, it was used to calculate the ranks from the entire, original $80 \%$

775 training dataset. The testing dataset then allowed us to test the efficiency of the calculated ranks

776 in predicting future aggressive outcomes. Here nTries $=449$ was chosen. 
David's Score

779 Pij represents the winning proportion of individual $\mathrm{i}$ against $\mathrm{j}$, which leads to a matrix of

780 observed win proportions as an output. For the Dij index, a matrix is obtained where the

781 observed proportion of wins (Pij) is corrected for the chance occurrence of this observed

782 outcome. Balasubramaniam et al. (2013) argued that Pij might be a better choice for species with

783 high levels of directional asymmetry (i.e., despotic species), whereas Dij may be a better choice

784 for species with low levels of directional asymmetry (i.e., tolerant species). We compared both

785 these indices. Furthermore, the David's score method can be calculated with the aid of three

786 different $\mathrm{R}$ packages. The decision was made to include them all in the analysis, producing two

787 calculated scores per package. This allowed us to assess whether the calculated ranks were the

788 same across all packages and if not, which package led to the highest percentage of accurate

789 predictions.

790

$791 \quad$ Percolation and Conductance

792 The parameter maxLength helps find all indirect pathways of a particular length and then update

793 the conflict matrix. Examining information gained through indirect pathways provides

794 information that can be used to decide on the appropriate maxLength for a dataset. To assist with

795 this decision, the Perc package offers a transitivity function as a way to estimate an alpha value,

796 which is used to weight the information from the indirect pathways to give an indication of the

797 extent to which we can trust information from indirect pathways. Greater transitivity is 
798 associated with assigning higher weight to information from indirect pathways (Fushing,

799 McAssey \& McCowan 2011). We tested MaxLengths of 2 and 4.

800 Elo-rating method

801 In the original method, $\mathrm{k}$ is held constant and all individuals receive the same elo-rating

802 at the initiation of the burn-in period. Elo-ratings were calculated with 1000 as the initial value

803 and $\mathrm{k}$ set to 100 (Neumann et al. 2011).

$805 \quad$ Modified Elo-rating (k variation)

806 In this method, each dominance interaction is classified according to the most intense

807 level of aggression displayed by the winner (Newton-Fisher 2017). This being required, we

808 excluded behaviours recorded as "unknown" from the analyses and distinguished among non-

809 aggressive, stationary, active and physical threats. This classification is based on our inter-troop

810 encounter protocol (Barrett, L. and Henzi, S.P., unpublished data). Non-aggressive behaviours

811 included supplants (i.e., where the aggressor takes the victim's place) and displacements (i.e.,

812 when one animal submissively moved away when approached within 10 meters). Any aggressive

813 behaviour that did not include a forward movement was considered a stationary threat, such as

814 lunge, facial and vocal threats. Active aggression involved ongoing forward movement (i.e.,

815 chase or charge) but where no physical contact was made with the target of aggression. Physical

816 aggression was scored in instances where body contact was made (e.g., a bite or slap). We

817 assigned a different $\mathrm{K}$ value to each of these categories, using the default value of 200 (Neumann

818 et al. 2011) for the most commonly observed form of aggression (i.e., the non-aggressive 
819 interactions of displace and supplant) and scaling up in multiples of 25 to distinguish varying

820 intensities. This led to the creation of a modified training data set and its detailed composition is

821 given in Table A1.

822

\begin{tabular}{|c|c|c|c|c|c|}
\hline & \multicolumn{5}{|c|}{ Modified training data set } \\
\hline Number & Total & Physical & Active & Stationary & Non-aggressive \\
\hline RBM Troop & 8083 & 886 & 1644 & 1625 & 3934 \\
\hline K value & - & 300 & 250 & 225 & 200 \\
\hline
\end{tabular}

823 Table A1: Modified training data set for the modified Elo-rating method (Newton-Fisher 2017).

824 Bayesian inference (BI) approach

825 We implemented this method Goffe, Fischer \& Sennhenn-Reulen's (2018) code (with no

826 additional analytical choices or justification required).

$827 \quad$ Randomized Elo-rating

By randomising the order of observed interactions, this approach allows the creation of $\mathrm{K}$

829 replicated datasets, where K corresponds to the number of randomisations performed. In this

830 study we were only interested in obtaining the final scores (return.as.ranks $=$ TRUE). The

831 function returns a NxK matrix that gives the final scores for each individual (rows) after each

832 randomisation of the order. In order to use the information contained in all the iterations, we

833 extracted the mean ranks for each individual. 
bioRxiv preprint doi: https://doi.org/10.1101/692384; this version posted August 27, 2019. The copyright holder for this preprint (which was not certified by peer review) is the author/funder, who has granted bioRxiv a license to display the preprint in perpetuity. It is made available under aCC-BY-NC-ND 4.0 International license.

834 We followed the same procedure as Sánchez-Tójar, Schroeder \& Farine (2018) and randomized 835 the order in which interactions occurred 1000 times. 